\title{
Effects of Histobulin on Depression and Anxiety in Chronic Urticaria: Psychiatric Manifestations or Psychiatric Comorbidities of Chronic Urticaria?: A Case Report
}

\section{G Noh ( $\square$ admyth@naver.com )}

Department of Allergy and Clinical Immunology https://orcid.org/0000-0002-4083-6844

\section{Sungjin Cho}

Cheju Halla General Hospital

\section{Case report}

Keywords: Histobulin, Immunotherapy, Chronic urticaria, Allergic psychiatric manifestation, histaminemediated psychiatric manifestation

Posted Date: October 22nd, 2020

DOl: https://doi.org/10.21203/rs.3.rs-94970/v1

License: (c) (1) This work is licensed under a Creative Commons Attribution 4.0 International License. Read Full License 


\section{Abstract}

Background: Psychiatric comorbidities of chronic urticaria (CU) have been reported and examined recently. The prevalence of mental disorders and emotional distress is high in patients with chronic urticaria. Histobulin is well known to be effective in chronic urticaria.

Case Presentation: Three cases of CU accompanying psychiatric manifestations (PMs) were treated with Histobulin. One patient with CU with severe depression showed clinical changes in depressive symptoms in parallel to changes in allergic symptoms. Histobulin improved not only chronic urticaria but also the accompanying PMs in two other patients with $\mathrm{CU}$. The PMs were not improved by an antihistamine (H1 blocker) in all 3 cases. Histobulin is effective not only for allergic manifestations (AMs) but also for PMs of chronic urticaria.

Conclusions: PMs seem to be clinical manifestations of chronic urticaria through histamine-mediated mechanisms. PMs as well as AMs of chronic urticaria were effectively and causatively treated with Histobulin. These conditions were suggested to be 'allergic psychiatric manifestations (APM)' or 'histamine-mediated psychiatric manifestations (HmPM). Further study of PMs based on histaminemediated mechanisms, including allergies, is necessary. Accordingly, it should be clarified whether the PMs of CU are actual PMs of CU or are psychiatric comorbidities of CU.

\section{Background}

HistobulinTM (Green Cross PD, Korea) is a histamine-fixed immunoglobulin preparation comprising 0.15 $\mu \mathrm{g}$ of histamine dihydrochloride and $12 \mathrm{mg}$ of $\operatorname{lgG}$ [1]. Histobulin is well known to be effective in chronic urticaria [2]. The prevalence of mental disorders and emotional distress is high in patients with chronic urticaria [3]. The improvement of psychiatric manifestations during Histobulin therapy for the treatment of chronic urticaria in three cases is described in this case report.

\section{Case Presentation}

\section{Case 1}

A 28-year-old female patient visited the Department of Allergy and Clinical Immunology, Cheju Halla General Hospital, due to eosinophilia. In the past, she suffered from atopic dermatitis, allergic rhinitis and chronic urticaria for 24 years and from severe depression with numerous suicide attempts for 14 years.

In the diagnosis of chronic urticaria, all patients showed urticaria, skin rash (including urticaria) and itching over 6 weeks. The symptoms and signs of chronic urticaria, such as skin rash, itching and urticaria, were well controlled by antihistamine (levocetrizine, a $\mathrm{H} 1$ receptor blocker) and did not show any positive findings in prior diagnostic examinations in other hospitals. According to the definition of chronic urticaria [4], the patients were diagnosed with chronic urticaria. 
Basic allergy tests (blood tests and skin prick tests) were conducted on all three patients before and after treatment. They underwent blood tests to determine the complete blood count with the differential count, serum eosinophil cationic protein, serum total IgE and IgE levels for specific allergens using a multiple allergosorbent test (MAST, Green Cross PD, Korea). In the MAST test, the specific IgEs for 41 allergens were evaluated, including Dermatophagoides pteronyssinus (Dp), Dermatophagoides farina (Df), cat, dog, egg white, milk, soybean, crab, shrimp, peach, mackerel, rye pollen, house dust mite, cockroach, Clasporium herbarum, Aspergillus fumigatus, Alternaria alternata, birch-alder mix, white oak, short ragweed, mugwort, Japanese hops, hazelnut, sweet vernal grass, Bermuda grass, orchard grass, timothy grass, reed, Penicillium notatum, sycamore, sallow willow, poplar mix, ash mix, pine, Japanese cedar, acacia, oxeye daisy, dandelion, Russian thistle, goldenrod and pigweed. The test results showed the level of specific IgE for each allergen, and a normal negative range was $0.000-0.349 \mathrm{IU} / \mathrm{mL}$.

A skin prick test was also performed for 53 allergens. The allergens tested by the skin prick test were Alternaria alternata, Aspergillus fumigatus, Aspergillus nigre, Candida albicans, Cladosporium, Penicillium chrysogenum, German cockroach, Dp, Df, dog, cat, grey elder/silver birch, grass mix, mugwort, short ragweed, black willow pollen, orchard grass, Bermuda grass, timothy grass, English plantain, English rye grass, Holm oak, Japanese cedar, cotton flock, milk mix, egg mix, chicken, beef, pork, cod, oyster, salmon, prawn, mackerel, tuna, almond, peanut, bean, carrot, cabbage, walnut, maize, peach, tomato, black pepper, spinach, wheat flour, rabbit, kapok, hops, acacia, pine and poplar. Skin prick tests were performed on the back of the patient. The area to be tested was cleaned with alcohol and coded with a skin marker corresponding to the number of allergens being tested. The marks were $2 \mathrm{~cm}$ apart. A drop of allergen solution was placed beside each mark. A small prick through the drop was made into the skin using a

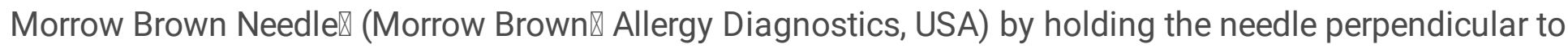
the test site and punching firmly through the tested extract and into the epidermis. The drop was removed immediately after the skin was pricked, and the used needle was discarded immediately. Histamine hydrochloride ( $1 \mathrm{mg} / \mathrm{ml}$ ) was used as a positive control, and physiologic saline was used as a negative control. The results were determined according to the wheal size. Reactions were read after $15 \mathrm{~min}$ and described as negative ( 0 , no reaction), $1+$ (reaction greater than the control reaction but smaller than half the size of the reaction to histamine), 2+ (equal to or more than half the size of the histamine reaction), $3+$ (equal to or more than the size of the histamine reaction) and 4+ (equal to or more than twice the size of the histamine reaction). The minimum size of a positive reaction was $3 \mathrm{~mm}$.

In the laboratory tests, the elevation of the eosinophil fraction based on the complete blood counts with differential counts was as high as $11.05 \%$ (normal $<=5 \%$ ), and the blood eosinophil cationic protein level was as high as $54.3 \mathrm{ng} / \mathrm{ml}$ (normal <= $24 \mathrm{ng} / \mathrm{ml}$ ). The serum total lgE level was as high as $3112 \mathrm{lU} / \mathrm{ml}$ (normal < = 350). According to the MAST, the positive allergens were Dp (>100), Df (>100), cat (76.75), Clasporium herbarum (0.56), Alternaria alternata (0.35), short Ragweed (0.48), mugwort (2.82), hazelnut (1.67), orchard grass (0.54), timothy grass (0.49), Penicillium notatum (0.65), sallow willow (26.85), Japanese cedar (7.60), oxeye daisy (8.14), dandelion (3.63) and goldenrod (11.23). The skin prick test results were positive for allergens of Dp (7/5), Df (10/6), dog (2/3), cat (5/4), mugwort (24/9), short 
ragweed (4/4), black willow (3/3), Japanese cedar (6/4), almond (3/3), bean (3/3), and histamine (7/5), and the normal control results were $0 / 0$.

The clinical progress of chronic urticaria was recorded as the frequency of allergic episodes, including the development of urticaria, skin rashes, and/or itching and the frequency of antihistamine intake.

Patients were evaluated before and after treatment to determine their mood using the Beck Depression Inventory (BDI)-2 for depression (normal $<=13$, mild $14-19$, moderate $20-28$, severe $29<=$ ) and the StateTrait Anxiety Inventory (STAl) for anxiety [5]

(state: normal < = 51, mild 52-56, moderate 57-61, severe 62<=; trait: normal < =53, mild 54-58, moderate $59-63$, severe $64<=)$. The patient in case 1 was the first case who presented symptoms and was the motivation for this study, and the BDI and STAI were not conducted. For the patient in the first case, the frequency of suicidal urges with depressive mood during one week was recorded as the 1st indicator for psychiatric evaluation.

Patients received $2 \mathrm{ml}$ of Histobulin ( $12 \mathrm{mg}$ human immunoglobulin/0.15 $\mu \mathrm{g}$ histamine complex) once a week by subcutaneous injection in the deltoid area of the upper arm. Patients were instructed to take a tablet of $5 \mathrm{mg}$ levocetrizine every day if necessary when they felt uncomfortable due to urticaria, skin rash or especially itching that prevented them from engaging in activities of normal daily living.

Before Histobulin treatment, allergic symptoms and signs related to chronic urticaria and allergic rhinitis occurred nearly every day. Depressive mood changes occurred, and the patient had suicidal urges every day. By using antihistamines, the allergic symptoms and signs were well controlled, but the psychiatric manifestations such as depressed mood and suicidal urges were not changed.

Histobulin therapy was attempted for the treatment of allergic rhinitis and chronic urticaria. Allergic rhinitis began to improve after 3 injections of Histobulin. Surprisingly, the frequency of suicidal urges also decreased simultaneously. Thereafter, the severity of the psychiatric manifestations were correlated with the severity of the allergic status (Fig. 1A). Consequently, after 26 injections of histobulin, the allergic rhinitis and chronic urticaria had remitted, and the concomitant depression and suicidal urges also disappeared.

\section{Case 2}

A 30-year-old female patient visited the Department of Allergy and Clinical Immunology, Cheju Halla General Hospital, due to itching and urticaria for 5 months. She was taking antihistamines daily and suffered from allergy symptoms and signs every day. There was no specific medical history, including a history of allergy or psychiatric problems. Angioedema occurred every week. She also indicated that the development of depression and anxiety coincided with the beginning of the allergic manifestations.

Her basophil fraction was as high as $1.1 \%$ (normal range: $0-1 \%$ ). The levels of serum eosinophil cationic protein and total IgE were normal. There were no positive results for the MAST and skin prick tests. The 
BDI score was 32 (severe), the STAI state score was 58 (moderate) and the STAI trait score was 56 (mild). According to the definition, her final diagnosis was chronic urticaria.

After the 3rd injection of Histobulin, the patient's allergic symptoms and signs improved along with the improvement of the psychiatric manifestations. Twelve weeks after injection of Histobulin, there were symptoms and signs, including urticaria and itching, as well as angioedema without antihistamine medication (Fig. 1B). According to the allergic laboratory test, the blood basophil fraction had become normal. Psychiatric manifestations were also absent after 12 weeks of treatment. The BDI score was 3 (normal). The STAI status score was 32 (normal), and the STAI trait score was 35 (normal).

\section{Case 3}

A 50-year-old female patient visited the Department of Allergy and Clinical Immunology, Cheju Halla General Hospital, due to suffering from itching and urticaria for 8 years. She was taking an antihistamine daily and was affected by allergy symptoms and signs every day. There was no specific medical history, including a history of allergy or psychiatric problems. She also experienced chest tightness with respiratory difficulty sometimes when the allergic manifestations appeared. She complained of both depression and anxiety.

Her laboratory tests were normal except for positive results for the MAST for Dp (4.27), Df (21.18) and shrimp (0.72). Additionally, there were no positive results for the skin prick test. The BDI score was 27 (moderate), the STAI state score was 62 (severe) and the STAI trait score was 52 (normal). According to the definition, her final diagnosis was chronic urticaria.

There were no changes in the clinical symptoms and signs of chronic urticaria or the frequency of antihistamine intake. However, after the 6th injection of Histobulin, the patient's psychological state was enhanced, with the improvement of depressive mood and anxiety. Twelve weeks after the injection of Histobulin, the chronic urticaria had not improved objectively (Fig. 1C). However, the anxiety was improved in spites of the aggravation of depression. The patient's BDI score was 38 (severe). The STAI status score was 57 (moderate) and the STAI trait score was 53 (normal) after treatment.

\section{Discussion And Conclusions}

Histobulin was effective for improving psychiatric manifestations in chronic urticaria (CU). The clinical results of this case report are as follows: 1) psychiatric manifestations (PMs) were improved by Histobulin therapy in all three cases regardless of the improvement of the allergic manifestations (AMs), and 2) interestingly, Histobulin therapy resulted in different results in each case. AMs and PMs were improved simultaneously in cases 1 and 2. However, only PMs were improved by Histobulin therapy without the improvement of AMs in case 3. The clinical responses in this report provide many important clues for better understanding the pathogenesis of PMs that are related to allergies and for new concepts for treatment modalities for psychiatric symptoms and signs. 


\section{Mechanisms of the effect of Histobulin on psychiatric manifestations in chronic urticaria}

PMs were clearly improved by Histobulin therapy in all 3 cases. In all three cases, AMs were well controlled with an antihistamine (H1 blocker) without the improvement of PMs before Histobulin therapy.

Histobulin treatment is a non-allergen-specific immunotherapy [6]. Histobulin inhibits mast cell degranulation and histamine release in rats [7]. These mechanisms are non-allergen-specific. Histobulin is a non-allergen-specific immunotherapy. One of the most important mechanisms of the effects of Histobulin is histaminopexy [8] and the production of antibodies against histamine [9]. Consequently, histaminopexy reduced the level of histamine; PMs in chronic urticaria are suspected to be histaminemediated symptoms and signs. Hereby, the use of the term 'histamine-mediated psychiatric manifestation (HmPM)' or 'allergic psychiatric manifestation (APM)' is suggested.

There are four kinds of histamine receptors [10]. $\mathrm{H} 1$ plays a well-known role in allergies. In general, antihistamines bind to the $\mathrm{H} 1$ receptor and resolves the allergic manifestations of chronic urticaria as well as other allergic diseases, including allergic rhinitis. Antihistamine $\mathrm{H} 1$ blocks the $\mathrm{H} 1$ receptor and interferes with the action of histamine through the $\mathrm{H} 1$ receptor [11]. Histaminopexy and the reduction of the systemic histamine level by Histobulin had the same effect on interfering with the action of histamine, regardless of the type of receptor (Fig. 2A). PMs did not seem to be improved by blocking only the $\mathrm{H} 1$ receptor and instead might be resolved by Histobulin, which might reduce the levels of histamines that affect all 4 histamine receptors on neurons (Fig. 2A). Through the effects of Histobulin on PMs in chronic urticaria, the effects of histamine on PMs in CU were demonstrated in this case report.

\section{Are histamine-mediated responses comorbidities or psychiatric manifestations of chronic urticaria?}

Psychiatric comorbidities have been the focus of recent reports, and there have been many reports concerning the relationship between allergic diseases and the manifestations and comorbidities of psychiatric disease [12]. Depression scores were positively correlated with changes in allergy symptoms in recurrent mood disorders after exposure to seasonal peaks in aeroallergens [13]. The relationship of high pollen counts and the seasonality of mood worsening was also reported [14]. Allergic rhinitis induces anxiety-like behaviour and altered social interactions in rodents [15]. In the 1 st case, the frequency of suicidal urges was evaluated according to a report that described allergy as a risk factor for suicide [16]. Based on the clinical progression of case 1, the psychiatric manifestations occurred in parallel to those of chronic urticaria during treatment, and psychiatric manifestations were affected by allergic provocation. This patient served as the initial motivation for this report.

The presence of psychiatric comorbidities is very important for determining the quality of life in chronic urticaria [17]. The treatment of psychiatric comorbidities as well as chronic urticaria is very important. It is very important to note that both AMs and PMs were treated effectively by Histobulin. The presence of 
histaminergic neurons that have $\mathrm{H} 1, \mathrm{H} 2, \mathrm{H} 3$, and $\mathrm{H} 4$ receptors strongly suggests the possibility of histamine-mediated neuropsychiatric responses without direct evidence. To date, it has not been confirmed whether psychiatric manifestations are independent of psychiatric comorbidities or are the clinical manifestations of chronic urticaria.

Histobulin therapy showed different results in the three cases. AMs and PMs were improved simultaneously in cases 1 and 2 . However, the anxiety was improved by Histobulin therapy without the improvement of AMs in case 3. The relationship between AMs and PMs that are psychiatric manifestations mediated by histamine has not been proven directly due to the absence of $\mathrm{H} 3$ and $\mathrm{H} 4$ blockers for therapeutic use as described above. Four possibilities may be suggested based on the results of this case report. 1) Psychiatric manifestations may be induced by chronic diseases, including chronic urticaria. This possibility could not be excluded in cases 1 and 2 . However, it is not applicable in case 3 (Fig. 2B). 2) Stressors such as allergy provocation in chronic urticaria also possibly induce psychiatric manifestations. This is also not applicable in case 3. 3) Psychiatric manifestations are comorbidities of chronic urticaria. Although psychiatric manifestations could be comorbidities of PMs in chronic urticaria in these cases, it is concluded that the psychiatric manifestations occur through histamine-mediated mechanisms, at least based on the fact that PMs in all these cases were resolved by Histobulin. Histobulin is effective for treatment of PMs in chronic urticaria. 4) Psychiatric manifestations are the clinical manifestations of chronic urticaria. It is very easy to explain the effects of Histobulin on PMs in chronic urticaria. Histobulin is effective for treatment of PMs, which is similar to the conclusion that PMs are comorbidities of CU. The last two mechanisms, 3) and 4), are applicable to all 3 cases. The PMs in chronic urticaria were strongly suspected to occur through histamine receptors in neurons.

The results of this case report indicate that psychiatric manifestations are the clinical manifestations of chronic urticaria, with the same histamine-mediated mechanisms, rather than comorbidities of allergic disease. Many reports indicate the possibility of this theory, and the results of this case report seem to be direct evidence that PMs are clinical manifestations of chronic urticaria as well as other allergic diseases rather than comorbidities of chronic urticaria. Based on these results, many PMs should be reviewed to determine whether histamine-mediated provocation of PMs was involved because some PMs might be resolved only by the treatment of histamine-mediated phenomena though allergen-specific immunotherapies or non-allergen-specific immunotherapies such as Histobulin.

In similar allergic conditions, not only do the kinds of allergic diseases but also the relevant symptoms and signs vary according to individual differences. Psychiatric manifestations may develop despite allergic provocations. Therefore, not all allergic patients show psychiatric manifestations, but some patients may develop only psychiatric manifestations due to histamine-mediated allergic conditions, such as allergy provocation or CU. Adult onset asthma due to mental disorders has been reported [18]. It is not clear whether the psychiatric manifestations or allergic symptoms and signs developed earlier or independently. According to the sensitivity of organs or individual differences, only histamine-mediated PMs possibly appeared without allergic disease. Namely, psychiatric conditions may develop without the presence of allergic diseases. Therefore, it is possible that patients are diagnosed with psychiatric 
conditions despite the allergic causes. Further clinical and experimental investigations are urgently needed of the mental health of humans according to the causative role of allergies in the provocation of PM.

'Conclusively, PM in chronic urticaria was improved by Histobulin. PMs were strongly suspected to be histamine-mediated clinical manifestations of $\mathrm{CU}$ rather than comorbidities of CU. Currently, Histobulin is the only effective therapeutic for the treatment of PMs as well as CU.

\section{List Of Abbreviations}

$\mathrm{CU}$, Chronic urticaria; AMs, Allergic manifestations; PMs, Psychiatric manifestations; Dp, Dermatophagoides pteronyssinus; Df, Dermatophagoides farina

\section{Declaration}

- Ethics approval and consent to participate

The IVIG therapy in this case was approved by the IRB of Cheju Halla General Hospital (IRB No. 2020-L0501).

- Consent for publication

Written informed consent was obtained from the patient for publication of this case report and any accompanying images. A copy of the written consent is available for review by the Editor of this journal.

- Availability of data and materials

Not applicable.

- Competing interests

The authors declare that they have no competing interests.

- Funding

None.

- Author's contributions

GN do major work for this report. SC manage the aspects of psychiatric evaluations. All authors have read and approved the manuscript.

\section{Acknowledgements}

\section{Contributor Information}


Geunwoong Noh. admyth@naver.com

Sungjin Cho. Elephant1234@gmail.com

\section{ORCID}

Geunwoong Noh, https://orcid.org/0000-0002-4083-6844

\section{References}

1. Kim JH, Shin IS, et al. Improved HPLC Method Using 2,3-naphthalenedicarboxaldehyde as Fluorescent Labeling Agent for Quantification of Histamine in Human Immunoglobulin Preparations. Osong public Health Res Perspect. 2011;2:127-34

2. Narayana J, Shianthi T, Bharadwaj S. Efficacy of histaglobulin on allergic rhinitis. Indian J Otolaryngol Head Neck Sug. 1997;49:77-9.

3. Staubach P, Dechene M, Metz M, et al. High prevalence of mental disorders and emiotional distress in patients with chronic spontaneous urticaria. Acta Derm Venereol. 2011;91:557-61.

4. Beck LA, Bernstein JA, Mauerer M. A Review of International Recommendations for the Diagnosis and Management of Chronic Urticaria. Acta Derm Venereol 2017;97:149-58.

5. D'Aurizio G, Caldarola A, Ninniri M et al. Sleep Quality and Psychological Status in a Group of Italian Prisoners. J Environ Res Public Health. 2020;17:4224.

6. Noh G. Immunotherapy Using Histobulin in Atopic Dermatitis. Clin Case Rep 2020 under review.

7. Ishikawa T, Shimada T, Kessoku N, et al. Inhibition of rat mast cell degranulation and histamine release by histamine-rat gammaglobulin conjugate. Int Arch Allergy Appl Immunol. 1979;59:403-7.

8. Parrot JL, Laborde $\mathrm{C}$. The histamine-fixing power of blood serum; its modification after an injection of normal human serum or of an azoprotein of histamine. J Physiol Paris. 1954;46:492-5.

9. Gushcin IS, Luss LV, Ilina NI, et al. Therapeutic effectiveness of histaglobin preparations in patients with allergic rhinitis and chronic urticaria. Ter Arkh. 1999;71:57-62.

10. Obara I, Telezhkin V, Alrashdi I, et al. Histamine, histamine receptors, and neuropathic pain relief. Br J Pharmacol. 2020;177:580-99.

11. Kawauchi H, Yanai K, Wang DY, et al. Antihistamines for allergic rhinitis treatment from the viewpoint of nonsedative properties. Int J Mol Sci. 2019;20:213.

12. Konstantinou GN, Konstantinou GN. Psychiatric comorbidity in chronic urticaria patients: a systematic review and meta-analysis. Clin Transl Allergy 2019;9:42.

13. Postolache TT, Lapidus M, Sander ER, et al. Changes in allergy symptoms and depression scores are positively correlated in patients with recurrent mood disorders exposed to seasonal peaks in aeroallergnes. Scientific World Journal. 2007;7:1968-77.

14. Guzman A, Tonelli LH, Roberts D, et al. Mood-worsening with high-pollen-counts and seasonality: a preliminary report. .J Affect Disord. 2007;101:269-74. 
15. Tonelli $\mathrm{LH}$, Katz $\mathrm{M}$, Kovacsics $\mathrm{CE}$, et al. Allergic rhinitis induces anxiety-like behavior and altered social interaction in rodents. Brain Behav Immun. 2009;23:784-93.

16. Postolache TT, Komarow H, Tonelli LH. Allergy: a risk factor for suicide? Curr Treat Options Neurol. 2008;10:363-76.

17. Ozkan M, Oflaz SB, Kocaman N, et al. Psychiatric morbidity and quality of life in patients with chronic idiopathic urticaria. Ann Allergy Asthma Immunol. 200;99:29-33.

18. Alonso J, de Jonge P, Lim CCW, et al. Association between mental disorders and subsequent adult onset asthma. J Psychiatr Res 2014;59:179-88.

\section{Figures}
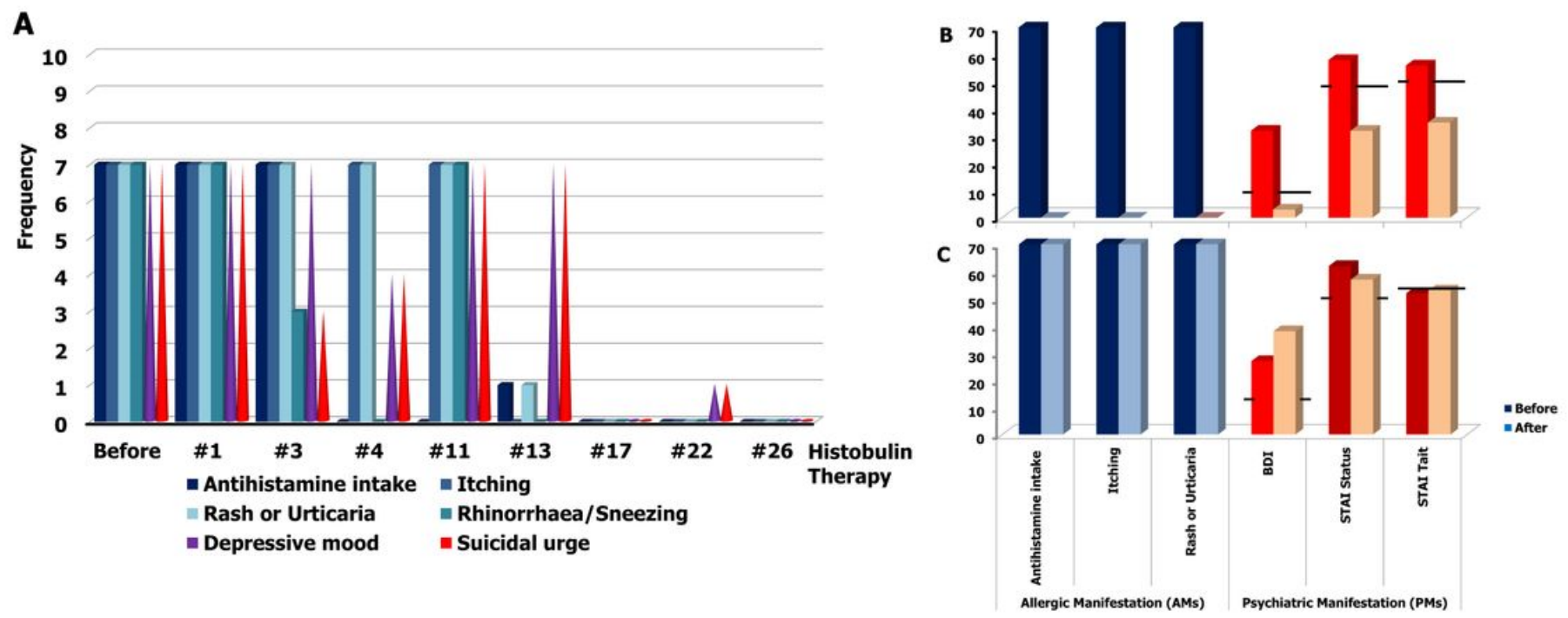

Figure 1

A. Clinical progress of case 1. After the 3rd injection of Histobulin, the patient showed the improvement of rhinorrhoea with a decrease in suicidal urges. Thereafter, allergic manifestations improved with a reduction in depressed mood and suicidal urges. Blue colour, allergic manifestations; red colour, psychiatric manifestations. B. Clinical results of case 2. AMs and PMs were all resolved after 12 injections of Histobulin. C. Clinical results of case 3. Only PMs were all resolved without the improvement of AMs after 12 injections of Histobulin. Black line, normal level. 
A

\section{H1 Receptor}

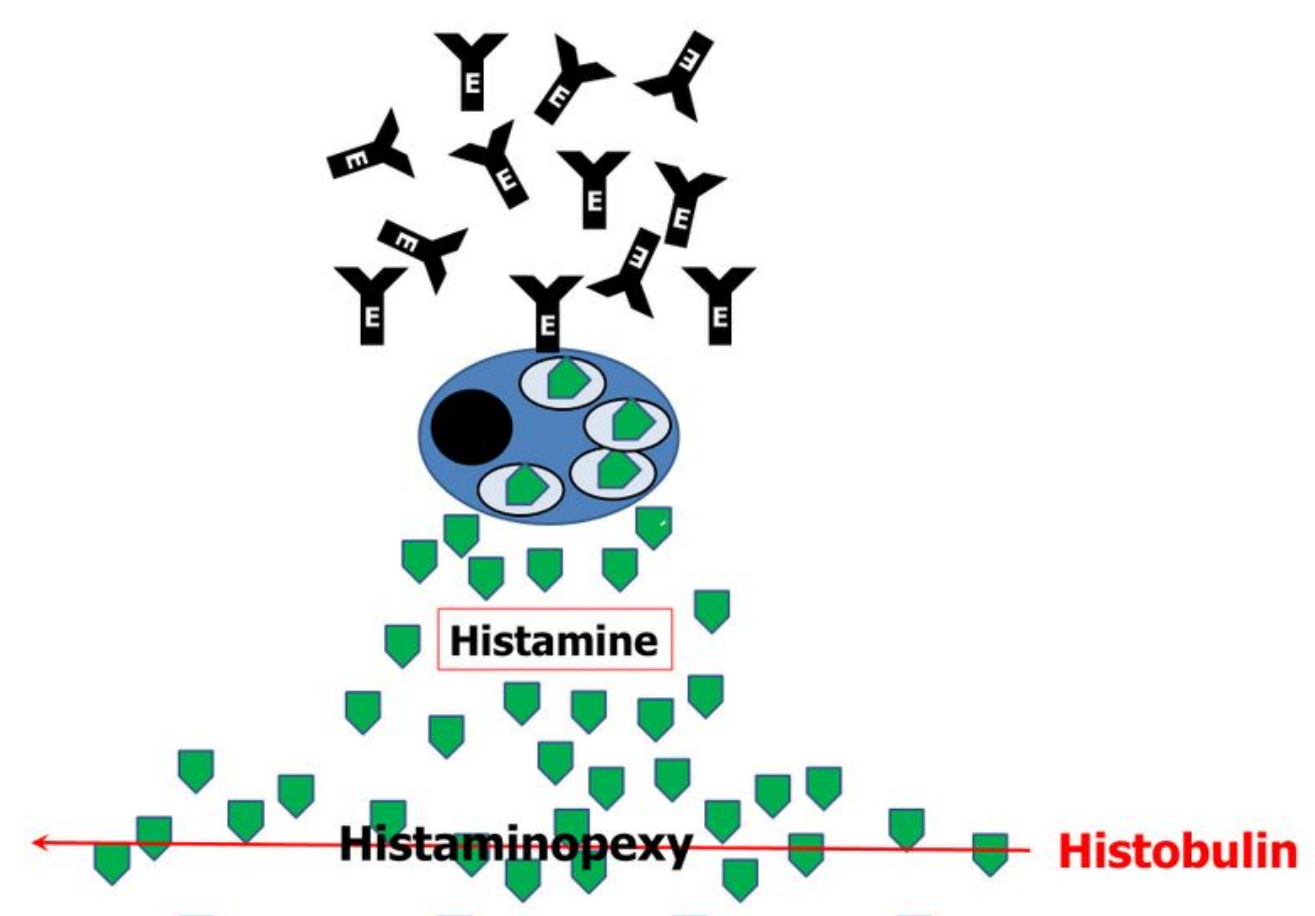

H2 Receptor
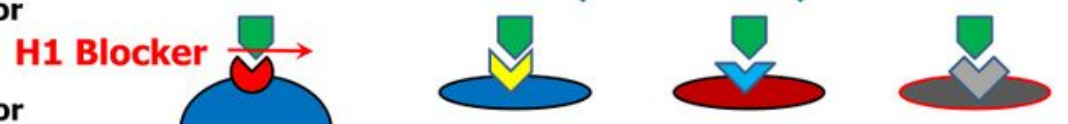

H3 Receptor

H4 Receptor

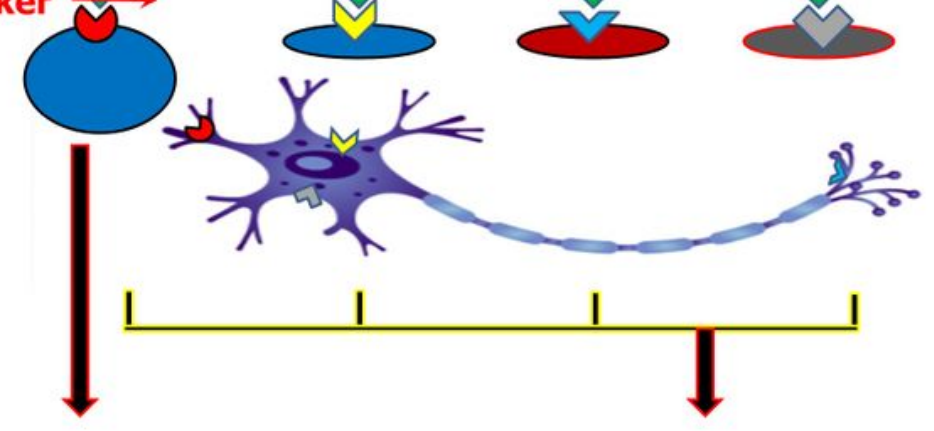

- Allergic Manifestations (AMs)

Urticaria

Angioedema

Anaphyalaxis

Respiratory Difficulty

- Psychiatric Manifestations (PMs)

Depression

Anxiety

Sleep disturbance

B

\begin{tabular}{|l|l|l|l|l|}
\hline Theory & Case $\mathbf{1}$ & Case $\mathbf{2}$ & Case $\mathbf{3}$ & Possible Thesis \\
\hline Chronic Disease -> PM & Possible & Possible & N/A & No \\
\hline Chronic Disease-> Stress Factor & Possible & Possible & N/A & No \\
\hline Comorbidity \& Histobulin Effects on PMs & Possible & Possible & Possible & Yes \\
\hline PMs as Histamine-mediated disease & Possible & Possible & Possible & Yes \\
\hline
\end{tabular}

\section{Figure 2}

A. The presumptive mechanisms of action of Histobulin. PMs were not changed by an antihistamine $\mathrm{H} 1$ blocker but were resolved by histamine. B. Investigation of the different responses of AMs and PMs in the 3 cases to Histobulin to rule out causes. Based on the different responses to Histobulin, 5 statuses are possible. 1) Psychiatric manifestations may be induced by chronic diseases, including chronic urticaria. 2) Stressors such as allergy provocation in chronic urticaria also possibly induce psychiatric 
manifestations. 3) Psychiatric manifestations are comorbidities of chronic urticaria. 4) Psychiatric manifestations are the clinical manifestations of chronic urticaria. The last two mechanisms, 3) and 4), are applicable to all 3 cases. Histobulin is effective in treating PMs in chronic urticaria regardless of whether they are psychiatric manifestations or psychiatric comorbidities of chronic urticaria. 